\title{
Systematic community- and hospital-based surveillance for enterovirus-D68 in three Canadian provinces, August to December 2014
}

DM Skowronski ${ }^{1}$, C Chambers $^{1}$, S Sabaiduc ${ }^{1}$, M Murti ${ }^{2}$, R Gustafson ${ }^{3}$, S Pollock ${ }^{4}$, D Hoyano ${ }^{5}$, S Rempel $^{26}$, S Allison ${ }^{7}, \mathbf{G}$ De Serres ${ }^{8}$, JA Dickinson ${ }^{9}$, R Tellier ${ }^{10}$, K Fonseca ${ }^{10}$, SJ Drews ${ }^{11}$, C Martineau ${ }^{8}$, F Reyes-Domingo ${ }^{6}, \mathrm{~T} \mathrm{Wong}^{12}{ }^{13}$, P Tang ${ }^{1}$, M Krajden ${ }^{1}$

1. 1. British Columbia Centre for Disease Control, Vancouver, British Columbia, Canada

2. Fraser Health Authority, Surrey, British Columbia, Canada

3. Vancouver Coastal Health Authority, Vancouver, British Columbia, Canada

4. Interior Health Authority, Kelowna, British Columbia, Canada

5. Vancouver Island Health Authority, Victoria, British Columbia, Canada

6. Public Health Agency of Canada, Ottawa, Ontario, Canada

7. Northern Health Authority, Prince George, British Columbia, Canada

8. Institut national de santé publique du Québec, Québec, Canada

9. University of Calgary, Alberta, Canada

10. Alberta Provincial Laboratory, Calgary, Alberta, Canada

11. Alberta Provincial Laboratory, Edmonton, Alberta, Canada

12. Health Canada, Ottawa, Ontario, Canada

13. Formerly affiliated with: Public Health Agency of Canada, Ottawa, Ontario, Canada

Correspondence: Danuta M Skowronski (danuta.skowronski@bccdc.ca)

Skowronski DM, Chambers C, Sabaiduc S, Murti M, Gustafson R, Pollock S, Hoyano D, Rempel S, Allison S, De Serres G, Dickinson JA, Tellier R, Fonseca K, Drews S), Martineau C, Reyes-Domingo F, Wong T, Tang P, Krajden M. Systematic community-and hospital-based surveillance for enterovirus-D68 in three Canadian provinces, August to December 2014. Euro Surveill. 2015;20(43):pii=30047. DOI: http://dx.doi.org/10.2807/1560-7917.ES.2015.20.43.300472015;20(34):p $\mathrm{ii}=30002$. DOl: $\mathrm{http}: / / \mathrm{dx}$.doi.org/10.2807/1560-7917.ES.2015.20.34.30002

Respiratory specimens collected from outpatients with influenza-like illness in three Canadian provinces (British Columbia (BC), Alberta and Quebec) participating in a community-based sentinel surveillance network were prospectively screened for enterovirusD68 (EV-D68) from 1 August to 31 December 2014 and compared to specimens collected from 1 October 2013 to 31 July 2014 . Eighteen (1\%) of 1,894 specimens were EV-D68-positive: $1 / 348$ (0.3\%) collected from October to December 2013 and 11/460 (2.4\%) from October to December 2014, an eight-fold increase in detection rates $(p=0.01)$, consistent with epidemic circulation in autumn 2014. The remaining EV-D68 detections were in September 2014 (6/37). Enhanced passive surveillance was also conducted on all inpatient and outpatient EV-D68 cases $(n=211)$ detected at the BC provincial reference laboratory from 28 August to 31 December 2014. Incidence of hospitalisations was $3 / 100,000$ overall and $21,17,4$ and 1/100,000 among those $\langle 5,5-9,10-19$ and $\geq 20$-years-old with male-tofemale ratios $>1$ among paediatric but not adult cases. Three cases in BC with comorbidity or co-infection died and five exhibited neurological features persisting $>9$ months. Active surveillance in outpatient and inpatient settings is needed from more areas and additional seasons to better understand EV-D68 epidemiology and potential at-risk groups for severe or unusual manifestations.

\section{Introduction}

Enterovirus-D68 (EV-D68) is a non-polio enterovirus that shares biological characteristics with enteroviruses (EVs) and rhinoviruses (RVs) [1]. First identified in California in 1962, EV-D68 was only sporadically detected in subsequent decades [2-4]. Between 2008 and mid-2014, however, EV-D68 was associated with several clusters of severe respiratory illness globally, disproportionately affecting children less than 5-yearsold [5-12].

In mid-August 2014, two paediatric hospitals in the United States (US) reported increases in severe EV-D68-associated respiratory illness [13] that were followed by a nationwide outbreak notable for affecting children with asthma in particular [14]. Several countries in Europe also reported EV-D68 activity during the summer and autumn of 2014, including one country, Norway, where EV-D68 was also associated with an increase in hospitalisations for severe respiratory illness [9,15-18]. In September 2014, the US Centers for Disease Control and Prevention (US CDC) additionally began investigating reports of acute flaccid myelitis of unknown aetiology in children, detecting EV-D68 in some, but not all, of these patients [19-22]; sporadic cases of EV-D68-associated neurological illness were also reported from France $(n=1)$ and Norway $(n=2)$ $[15,23,24]$. 


\section{FIGURE 1}

Epidemic curve of EV/RV, EV-D68 and influenza detections by month of specimen collection, Canadian Sentinel Practitioner Surveillance Network, British Columbia, Alberta and Quebec, 1 October 2013-31 December 2014 (n=1,909)

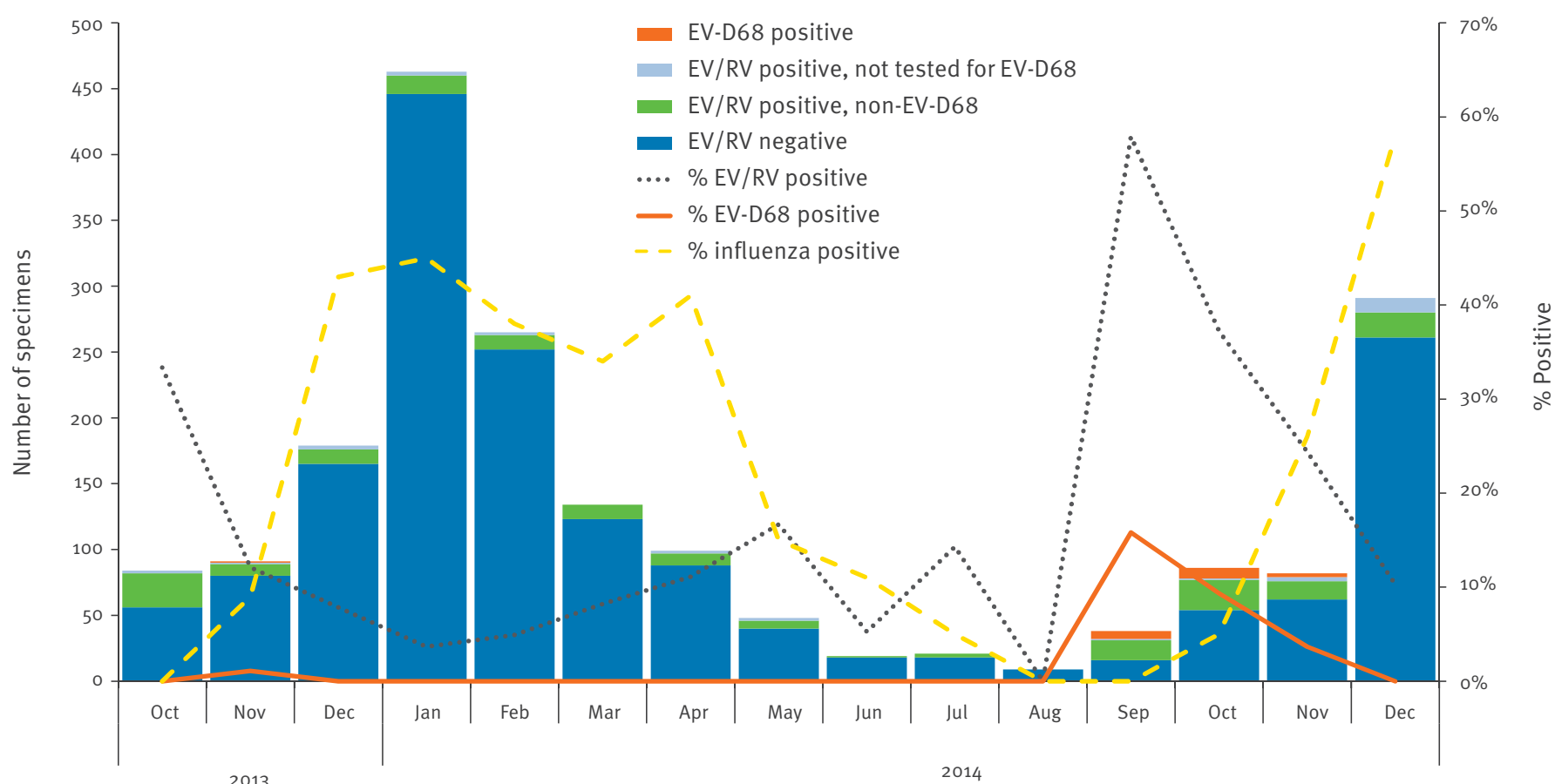

Month of specimen collection

EV: enterovirus; RV: rhinovirus; RVP: Respiratory Virus Panel.

a Excludes 169 specimens not tested for EV/RV: 133 influenza-positive specimens from Alberta not tested by Luminex xTAG RVP during $2014 / 15$ season, 20 specimens with insufficient viral load for testing, and 16 specimens from British Columbia that were tested only for EV-D68 (not other EV/RV) using an EV-D68-specific PCR assay (all EV-D68 negative), as per protocol indicated in Table 1.

\section{FIGURE 2}

Epidemic curve by week of specimen collection and hospitalisation status, laboratory-based enhanced passive surveillance, British Columbia, 28 August-31 December 2014 ( $\mathrm{n}=211)$

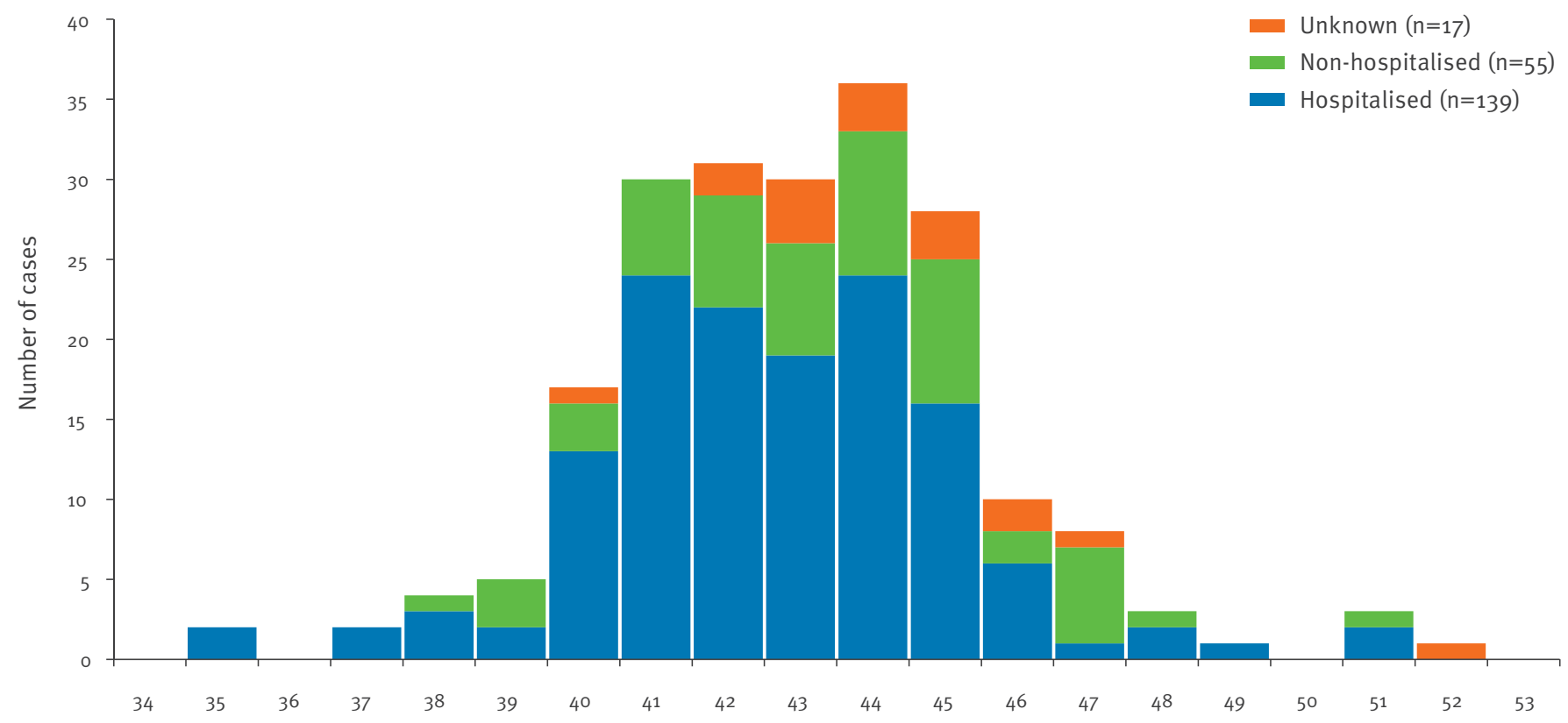

Week of specimen collection (2014) 
Overview of active community-based sentinel surveillance and laboratory-based enhanced passive surveillance used for EVD68 detection and characterisation, Canada, 2014

\begin{tabular}{|c|c|c|}
\hline Attribute & Canadian Sentinel Practitioner Surveillance Network & $\begin{array}{l}\text { British Columbia Public Health Microbiology and } \\
\text { Reference Laboratory }\end{array}$ \\
\hline Type of surveillance & Active, community-based sentinel & Enhanced passive, laboratory-based \\
\hline Setting & Outpatient sentinel sites & $\begin{array}{l}\text { Inpatient and outpatient specimens } \\
\text { submitted to BC PHMRL }\end{array}$ \\
\hline Province(s) & BC, Alberta, Quebec & $\mathrm{BC}$ \\
\hline Time period ${ }^{\text {a }}$ & 1 August-31 December 2014 & 28 August-31 December $2014^{\text {b }}$ \\
\hline Period of comparison ${ }^{\text {a }}$ & 1 October 2013-31 July 2014 & None \\
\hline Patient population & $\begin{array}{l}\text { Patients presenting within seven days of ILIc onset to } \\
\text { sentinel practitioners }\end{array}$ & $\begin{array}{l}\text { All laboratory-confirmed cases of EV-D68 in BC } \\
\text { residents } \\
\text { Age restrictions for testing: } \\
9 \text { Sep 2014-18 Sep 2014: } \\
\text { Inpatients: } 55 \text { years old } \\
\text { Outpatients: <5 years old } \\
19 \text { Sep 2014-20 Oct 2014: } \\
\text { Inpatients: no age restriction } \\
\text { Outpatients: }<20 \text { years old } \\
21 \text { Oct 2014-31 Dec } 2014: \\
\text { Inpatients: no age restriction Outpatients: no age } \\
\text { restriction }\end{array}$ \\
\hline Source of data & $\begin{array}{l}\text { Patient/provider self-report; standard questionnaire } \\
\text { completed at time of specimen collection }\end{array}$ & $\begin{array}{l}\text { Patient/provider self-report and/or electronic } \\
\text { medical record; enhanced surveillance form } \\
\text { completed at case detection }\end{array}$ \\
\hline Specimen type & Respiratory (nasal/nasopharyngeal) & Respiratory at clinician discretion \\
\hline Laboratory testing & $\begin{array}{l}\text { All provinces: EV/RV screening by Luminex xTAG RVP } \\
\text { BC PHMRL: EV-specific [31] and/or EV-D68-specific RT-PCR } \\
\text { assay and partial VP1 sequencing [10], as per BC PHMRL } \\
\text { protocol } \\
\text { Albertae: EV-specific PCR assay [27] and VP4/partial VP2 } \\
\text { sequencing [28-30] } \\
\text { Quebec: Partial VP1 sequencing [32] }\end{array}$ & $\begin{array}{l}9 \text { Sep 2014-20 Oct 2014: Luminex xTAG RVP and } \\
\text { partial 5' UTR/VP1 sequencing [10] } \\
21 \text { Oct 2014-13 Nov 2014: EV-specific RT-PCR assay } \\
\text { [31], EV-D68-specific RT-PCR assay and partial VP1 } \\
\text { sequencing [10] } \\
14 \text { Nov 2014-31 Dec 2014: EV-D68-specific RT-PCR } \\
\text { assay and partial VP1 sequencing [10] }\end{array}$ \\
\hline
\end{tabular}

BC: British Columbia; EV: enterovirus; ILI: influenza-like illness; PHMRL: Public Health Microbiology and Reference Laboratory; RVP: Respiratory Virus Panel; RV: rhinovirus; SPSN: Sentinel Practitioner Surveillance Network.

a Defined using specimen collection date.

${ }^{b}$ Full enhanced surveillance case report form completed until 31 October 2014; partial enhanced surveillance data (basic demographic data and hospitalisation status) collected until 31 December 2014.

' Defined as acute respiratory illness with fever and cough and at least one of sore throat, arthralgia, myalgia or prostration.

${ }^{d}$ EV-D68 cases in out-of-province residents, sentinel patients detected through the Canadian SPSN, and those with unknown/missing geographic information were excluded from enhanced passive surveillance analysis.

e EV/RV screening by Luminex xTAG RVP not performed on influenza-positive specimens collected during $2014 / 15$ influenza season (1 October 2014-31 December 2014) in Alberta.

EV-D68 infection is not generally notifiable and laboratory testing for EV-D68 was not widely performed prior to the 2014 outbreak. Consequently, localised clusters of paediatric hospitalisations have largely shaped current understanding of EV-D68 illness, skewing impressions of typical disease severity. Few countries have utilised existing general practice sentinel surveillance schemes for influenza-like illness (ILI) or acute respiratory illness (ARI) to systematically assess outpatient illness due to EV-D68 infection, as conducted in the Netherlands and Germany $[8,9,18]$.

In Canada, pre-existing infrastructure for standardised screening of respiratory specimens was mobilised in response to the alerts from the US. Respiratory specimens collected from ILI patients of all ages attending sentinel outpatient practices in three participating provinces (British Columbia (BC) (population:
4.4 million [25]), Alberta (population: 3.6 million [25]) and Quebec (population: 7.9 million [25])) of the community-based Canadian Sentinel Practitioner Surveillance Network (SPSN) were retrospectively and prospectively screened for EV-D68 before and during the 2014 August-to-December epidemic period. In addition, laboratory-based enhanced passive surveillance was conducted in BC on all detections of EV-D68 from inpatient and outpatient specimens tested at the $B C$ Public Health Microbiology and Reference Laboratory (BC PHMRL) during the 2014 epidemic period. Here we report findings from these two surveillance approaches to inform the epidemiology of EV-D68 including the spectrum of illness, population-based incidence, and potential at-risk groups for severe or unusual sequelae, including neurological or fatal outcomes. 


\section{FIGURE 3}

Phylogenetic analysis of EV-D68 partial VP1 sequences, enhanced passive surveillance (British Columbia), 28 August-31 December 2014, and Canadian Sentinel Practitioner Surveillance Network (British Columbia and Quebec), 1 October 2013-31 December 2014

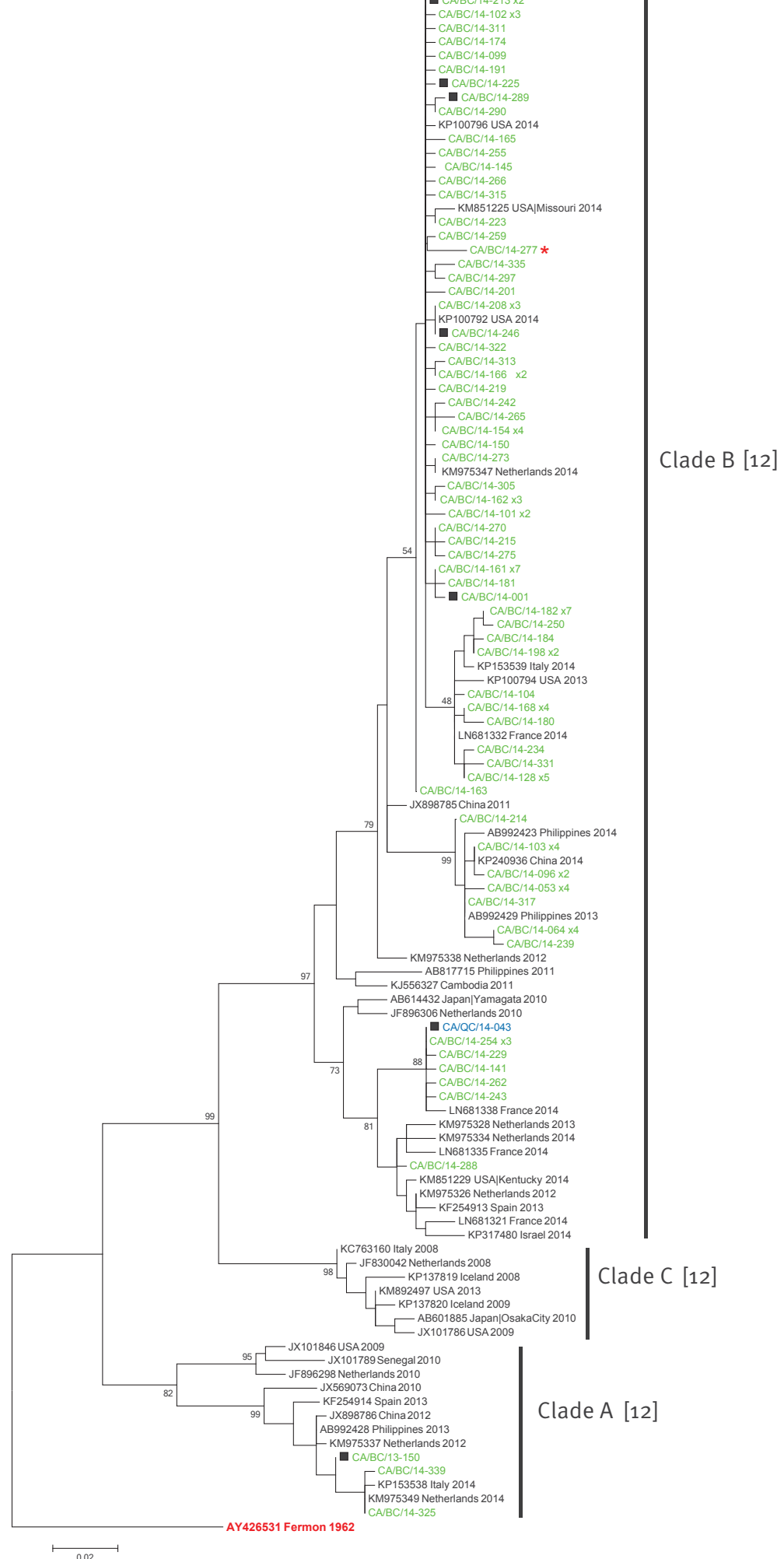

Canadian EV-D68 sequences are colour coded by province of specimen collection, global sequences available in GenBank are shown in black and the Fermon reference strain (1962) is shown in red. Sentinel viruses collected through the Canadian Sentinel Practitioner Surveillance Network are indicated with black squares. Red asterisks denote sequences with adjacent asparagine deletions at positions 144-145 in the D-E immunogenic loop.

Phylogenetic clusters are labelled clade A, B, and C as described in Tokarz et al. [12], corresponding to major group 3, 1, and 2 as described in Meijer et al. [9] and lineage 2, sub-lineage 1.2, and sub-lineage 1.1 as described in Lauinger et al. [7], respectively. 


\section{FIGURE 4}

Phylogenetic analysis of EV-D68 VP4/partial VP2 sequences, Canadian Sentinel Practitioner Surveillance Network (Alberta), 1 October 2013-31 December 2014

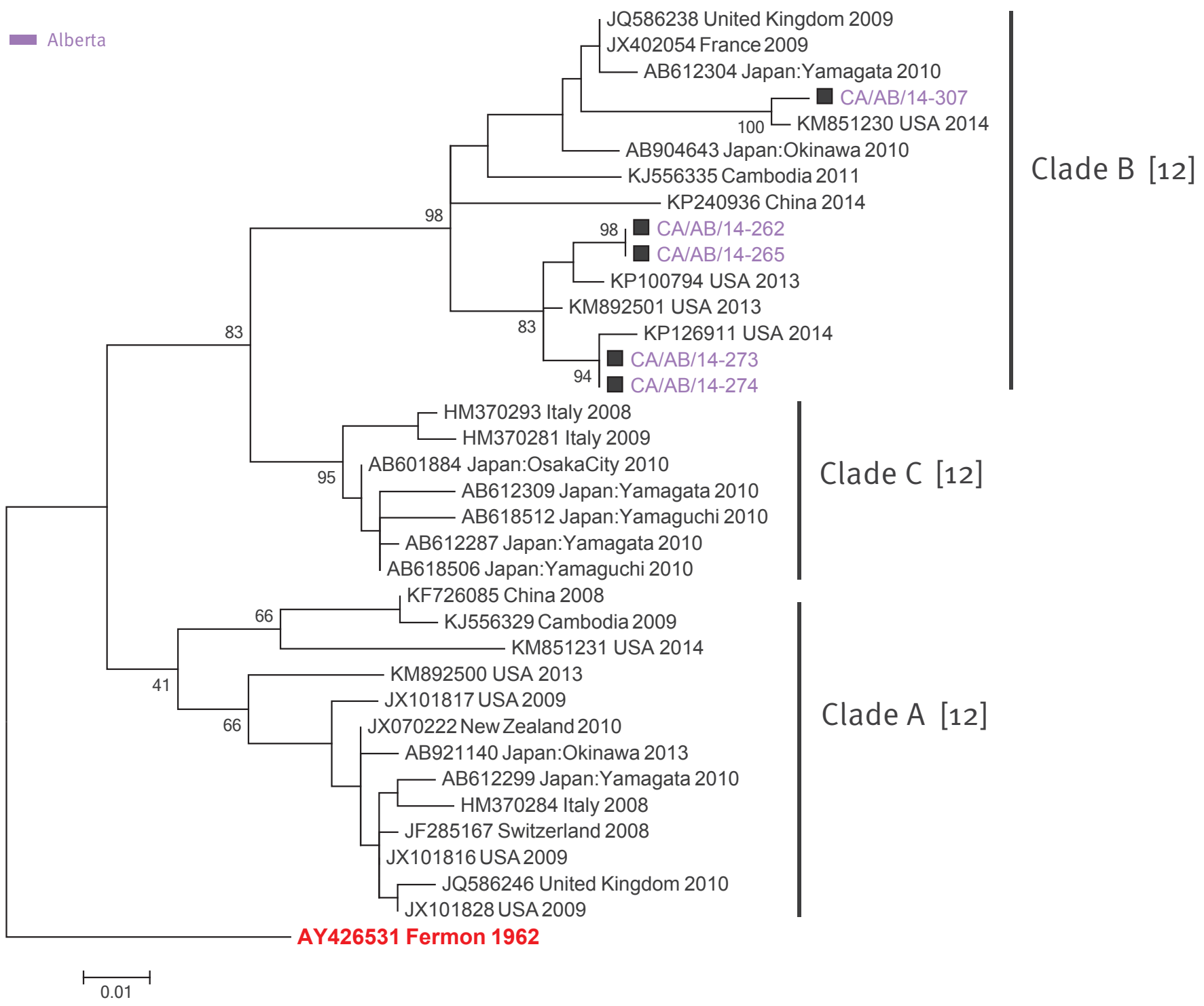

Canadian EV-D68 sequences are colour coded by province of specimen collection, global sequences available in GenBank are shown in black and the Fermon reference strain (1962) is shown in red. Sentinel viruses collected through the Canadian Sentinel Practitioner Surveillance Network are indicated with black squares.

Phylogenetic clusters are labelled clade A, B, and C as described in Tokarz et al. [12], corresponding to major group 3, 1 , and 2 as described in Meijer et al. [9] and lineage 2, sub-lineage 1.2, and sub-lineage 1.1 as described in Lauinger et al. [7], respectively.

\section{Methods}

Epidemiological sampling and laboratory testing protocols are outlined in Table 1 and are summarised below.

Active community-based sentinel surveillance Community-based sentinel practitioners of the Canadian SPSN collect nasal or nasopharyngeal specimens year-round from patients presenting within seven days of ILI onset defined as acute respiratory illness with fever and cough and at least one of the following symptoms: sore throat, arthralgia, myalgia or prostration [26]. Epidemiological information is collected from consenting patients/guardians using a standard questionnaire at the time of specimen collection. Research ethics board (REB) approval is obtained in each participating province.

The public health laboratories in the provinces of BC, Alberta and Quebec conducted prospective EV-D68 testing of EV/RV-positive SPSN specimens collected between 1 August and 31 December 2014. Additionally, they retrospectively tested specimens collected between 1 October 2013 and 31 July 2014 that were EV/RV-positive. The latter period was chosen as comparison because routine SPSN influenza surveillance 
TABLE 2

Characteristics of patients with specimens collected by the Canadian Sentinel Practitioner Surveillance Network and tested for EV-D68, British Columbia, Alberta and Quebec, 1 August-31 December $2014(n=506)$

\begin{tabular}{|c|c|c|}
\hline Characteristic, n (\% by column) & $\begin{array}{l}\text { EV-D68- } \\
\text { positive }^{\mathrm{a}}\end{array}$ & $\begin{array}{l}\text { EV-D68- } \\
\text { negative }\end{array}$ \\
\hline $\mathrm{N}^{\mathrm{b}}$ (\% by row) & $17(3)$ & $489(97)$ \\
\hline \multicolumn{3}{|l|}{ Age group (years) } \\
\hline$<5$ & 1 & $42(9)$ \\
\hline $5-9$ & 2 & $35(7)$ \\
\hline $10-19$ & 3 & $61(12)$ \\
\hline $20-59$ & 10 & $260(53)$ \\
\hline$\geq 60$ & 1 & $90(18)$ \\
\hline Unknown & 0 & $1(0)$ \\
\hline Median (range) & $23(1-64)$ & $36(0-94)$ \\
\hline \multicolumn{3}{|l|}{ Sex } \\
\hline Male & 10 & $192(39)$ \\
\hline Paediatric («20 years) & 2 & $53(28)$ \\
\hline Adult ( $\geq 20$ years) & 8 & $138(72)$ \\
\hline Female & 7 & $295(60)$ \\
\hline Paediatric ( $<20$ years) & 4 & $84(28)$ \\
\hline Adult ( $\geq 20$ years) & 3 & $211(72)$ \\
\hline Unknown & 0 & $2(0)$ \\
\hline \multicolumn{3}{|c|}{ Specimen collection interval (days)c } \\
\hline$\leq 4$ & 12 & $345(71)$ \\
\hline $5-7$ & 5 & $144(29)$ \\
\hline Median (range) & $4(0-7)$ & $3(0-7)$ \\
\hline \multicolumn{3}{|l|}{ Chronic comorbidity } \\
\hline No & 15 & $335(69)$ \\
\hline Yes & 1 & $117(24)$ \\
\hline Unknown & 1 & $37(8)$ \\
\hline \multicolumn{3}{|l|}{ Province } \\
\hline$B C$ & 10 & $126(26)$ \\
\hline Alberta & 5 & $177(36)$ \\
\hline Quebec & 2 & $186(38)$ \\
\hline
\end{tabular}

All values are number (n) and percentage (\%) by column where displayed (except in initial header row), unless otherwise specified.

$B C$ : British Columbia; EV: enterovirus; RV: rhinovirus.

a One EV-D68 detection in a specimen collected from an adult in British Columbia in November 2013 is not included in this table which is restricted to specimens collected during the 2014 epidemic period.

${ }^{b}$ EV-D68 typing was not performed on 16 specimens with insufficient viral load; 16 BC specimens were tested only for EV-D68 (not other EV/RV) using an EV-D68-specific PCR assay as per protocol in Table 1.

Interval between onset of influenza-like illness and collection of nasal/nasopharyngeal specimen.

typically commences on 1 October each year. Although the SPSN is primarily designed for influenza surveillance, public health laboratories in these provinces also routinely test SPSN specimens for a panel of respiratory viruses, including $E V / R V$, influenza $A\left(H_{1}\right.$, $\mathrm{H}_{3}$ and $\mathrm{H}_{1} \mathrm{~N}_{1}$ pdmog subtypes), influenza $\mathrm{B}$, respiratory syncytial virus, parainfluenza types $1-4$, human metapneumovirus, adenovirus, coronaviruses NL63, HKU1, 229E and OC43, and bocavirus (BC and Quebec only) using versions of the Luminex XTAG Respiratory Virus Panel (RVP) (Luminex Corp., US). During the 2014/15 influenza season, Alberta made the a priori decision to restrict routine EV/RV testing to influenzanegative patients for resource reasons.

Provincial protocols for EV-D68 detection among EV/ RV-positive specimens are specified in Table $1[10,27$ 32]. These assays have comparable sensitivities and limits of detection are considered within one $\log _{10}$ copy $/ \mathrm{mL}$, as demonstrated in a national validation study [33]. Specific EV-D68 typing was established by partial sequencing of structural capsid viral protein (VP) namely $\mathrm{VP}_{1}$ in $\mathrm{BC}$ [10] and Quebec [32] and VP2 in Alberta [28-30].

Laboratory-based enhanced passive surveillance in British Columbia

The BC PHMRL is the only site to provide confirmatory diagnosis of EV-D68 in BC. Such testing is usually conducted upon physician request; until 19 September 2014, this was predominantly to confirm diagnosis among inpatients at the province's tertiary paediatric hospital. From 9 September 2014 onwards, this also included routine screening of all respiratory specimens collected from inpatients or outpatients less than 5-years-old. On 19 September 2014, routine EV-D68 screening was extended to all respiratory specimens submitted to the BC PHMRL from all inpatients of any age across the province. Outpatient specimens were also included in routine EV-D68 screening with staggered implementation as shown in Table 1. EV-D68 typing was performed as specified in Table $1[10,31]$.

Detailed case report forms were completed on all EV-D68 detections by the BC PHMRL for specimens collected between 28 August and 31 October 2014, with only basic demographic and hospitalisation status recorded thereafter to 31 December 2014. Forms were completed by local public health practitioners based primarily upon the electronic medical record and reported to the $B C$ Centre for Disease Control (BCCDC) as part of outbreak investigation, exempt from REB approval. Patients presenting with neurological or fatal outcome notified to BCCDC as part of enhanced passive surveillance are further described as a case series based upon information in the electronic medical record and supplemented by direct patient, guardian and/or clinician interview.

\section{Phylogenetic analyses}

Partial VP1 sequences (nucleotides 133-471), including $B-C$ and $D-E$ immunogenic loops, from BC and Quebec were aligned to a subset of representative VP1 sequences in GenBank to establish clade designation $[2,12,34]$. Since no recombination was observed within $\mathrm{VP}_{4}$, the complete VP4 and partial VP2 (first 215 nucleotides of 5 ' end) sequences from Alberta were used to align with $\mathrm{VP}_{4} / \mathrm{VP}_{2}$ sequences in GenBank and divided 
TABLE 3

Characteristics of EV-D68 cases with full case report forms completed, laboratory-based enhanced passive surveillance, British Columbia, 28 August-31 October $2014(\mathrm{n}=146)$

Characteristic, $n$ (\% by column)

\begin{tabular}{l|l|l|l|l|l|l} 
Overall $^{\mathrm{a}}$ & All cases & Paediatric ( $(20$ years) & Adult ( $\geq 20$ years) & All cases & Paediatric ( $(20$ years) & Adult ( $\geq 20$ years)
\end{tabular}

\begin{tabular}{|c|c|c|c|c|c|c|c|}
\hline $\mathrm{N}$ (\% by row) & 146 & 111 & $91(82)$ & $20(18)$ & 35 & $26(74)$ & $9(26)$ \\
\hline \multicolumn{8}{|l|}{ Age group (years) } \\
\hline$<5$ & $53^{b}(36)$ & $39(35)$ & $39^{c}(43)$ & NA & 14 & $14^{\mathrm{d}}$ & NA \\
\hline $5-9$ & $39(27)$ & $32(29)$ & $32(35)$ & NA & 7 & 7 & NA \\
\hline $10-19$ & $25(17)$ & $20(18)$ & $20(22)$ & NA & 5 & 5 & NA \\
\hline$\geq 20$ & $29(20)$ & $20(18)$ & NA & 20 & 9 & NA & 9 \\
\hline Median age (range) & $\begin{array}{c}8 \\
(0-90)\end{array}$ & $8(0-82)$ & $5(0-19)$ & $35.5(21-82)$ & $7(0-90)$ & $4(0-19)$ & $42(20-90)$ \\
\hline \multicolumn{8}{|l|}{ Sex } \\
\hline Male & $85(58)$ & $65(59)$ & $59(65)$ & 6 & 20 & 18 & 2 \\
\hline Female & $61(42)$ & $46(41)$ & $32(35)$ & 14 & 15 & 8 & 7 \\
\hline \multicolumn{8}{|c|}{ Month of specimen collection } \\
\hline August & $2(1)$ & $2(2)$ & $2(2)$ & 0 & 0 & 0 & 0 \\
\hline September & $19(13)$ & $14(13)$ & $14(15)$ & 0 & 5 & 5 & 0 \\
\hline October & $125(86)$ & $95(86)$ & $75(82)$ & 20 & 30 & 21 & 9 \\
\hline $\begin{array}{l}\text { Median (range) } \\
\text { hospital stay (days) }\end{array}$ & NA & $3(1-18)$ & $3(1-18)$ & $4(1-12)$ & NA & NA & NA \\
\hline \multicolumn{8}{|l|}{ Admitted to ICU } \\
\hline Yes & NA & $9(8)$ & $7(8)$ & 2 & NA & NA & NA \\
\hline No & NA & $87(78)$ & $71(78)$ & 16 & NA & NA & NA \\
\hline Unknown & NA & $15(14)$ & $13(14)$ & 2 & NA & NA & NA \\
\hline \multicolumn{8}{|l|}{ Clinical presentation $^{f}$} \\
\hline Respiratory illness & $135(92)$ & $103(93)$ & $83(91)$ & 20 & 32 & 24 & 8 \\
\hline Pneumonia diagnosis & $27(18)$ & $25(23)$ & $19(21)$ & 6 & 2 & 2 & 0 \\
\hline Neurological illness ${ }^{g}$ & $4(3)$ & $4(4)$ & $4(4)$ & 0 & 0 & 0 & 0 \\
\hline Immunocompromised ${ }^{f}$ & $4(3)$ & $4(4)$ & $3(3)$ & 1 & 0 & 0 & 0 \\
\hline $\begin{array}{l}\text { Travel outside } \\
\text { Canada }{ }^{f, h}\end{array}$ & $5(3)$ & $4(4)$ & $4(4)$ & 0 & 1 & 1 & 0 \\
\hline Co-infection $^{f, i}$ & $10(7)$ & $10(9)$ & $9(10)$ & 1 & 0 & 0 & 0 \\
\hline Asthma prevalence ${ }^{f}$ & $55(38)$ & $47(42)$ & $39(43)$ & 8 & 8 & 5 & 3 \\
\hline Among males & $33(39)$ & $31(48)$ & $28(47)$ & 3 & 2 & 2 & 0 \\
\hline Among females & $22(36)$ & $16(35)$ & $11(34)$ & 5 & 6 & 3 & 3 \\
\hline
\end{tabular}

All values are number (n) and percentage (\%) by column where displayed (except in initial header row), unless otherwise indicated.

ICU: intensive care unit; NA: not applicable.

${ }^{a}$ Restricted to non-sentinel cases in British Columbia residents with enhanced surveillance case report forms with valid hospitalisation information collected as at 31 October 2014. Six cases missing information on hospitalisation status were excluded.

${ }^{b}$ Includes 14 infant cases 11 year-old and 20 cases 1-2-years-old (i.e. 34 cases «3-years-old).

c Includes nine hospitalised infant cases $<1$ year-old and 16 hospitalised cases $1-2$ years-old (i.e. 25 cases $<3$-years-old).

${ }^{d}$ Includes five non-hospitalised infant cases $\ll 1$ year-old and four non-hospitalised cases 1-2 years-old (i.e. nine cases $<3$-years-old).

e Five patients remained in hospital at time of reporting and were excluded from length of stay calculations.

${ }^{f}$ Proportions displayed are among those with known information only.

${ }^{\mathrm{g}}$ One adult case with neurological illness with specimen collection in November 2014 not included in this table which is restricted to specimens collected from 28 August to 31 October 2014 with full enhanced surveillance case report forms.

${ }^{\mathrm{h}}$ In 30 days prior to symptom onset; all five patients reported travel to the United States.

influenza $\mathrm{A}\left(\mathrm{H}_{3} \mathrm{~N}_{2}\right)(\mathrm{n}=1)$, respiratory syncytial virus $(\mathrm{n}=1)$, and Streptococcus pneumoniae $(\mathrm{n}=8$, including one detection in blood and seven in upper respiratory specimen). 
into clades corresponding to VP1 designations [12]. Phylogenetic trees were constructed in MEGA6, rooted to the 1962 prototype Fermon strain using the maximum-likelihood method with a Jukes-Cantor substitution model and 1,000 bootstrap replicates [35].

\section{Results}

Active community-based sentinel surveillance Among 2,078 specimens submitted to the Canadian SPSN, meeting ILI criteria and collected between 1 October 2013 and 31 December 2014, 1,909 (92\%) were screened for EV/RV and 221 (12\%) tested EV/ RV-positive. There was a mirror-image pattern of EV/ $R V$ versus influenza test-positivity by month, reflecting their differences in seasonality (Figure 1).

During this period, 1,894 of $2,078(91 \%)$ specimens were assessed for EV-D68, of which 18 (1\%) tested positive. This includes 1 of $348(0.3 \%)$ collected from October to December 2013 and 11 of 460 (2.4\%) from October to December 2014, a significant eight-fold increase in detection rates across two successive years (Chi-square test $\mathrm{p}=0.01$ ). The remaining EV-D68 detections were from specimens collected in September 2014 (6/37) (Figure 1). There were no EV-D68 co-infections with other respiratory viruses included on the Luminex xTAG RVP panel.

Six of the 17 EV-D68-positive cases detected during the 2014 epidemic period were $<20$-years-old. Detection rates among adults 20 to 59 -years-old (10/270; 4\%) did not differ from rates among children 20 -yearsold $(6 / 144 ; 4 \%)$. A single EV-D68 case was detected among adult patients $\geq 60$-years old $(1 / 91 ; 1 \%)$. Two of six paediatric EV-D68 cases were male compared to 8 of 11 adult cases. Other patient characteristics among EV-D68 test-positive and test-negative specimens for the August-to-December 2014 epidemic period are shown in Table 2.

Laboratory-based enhanced passive surveillance in British Columbia

Of 3,716 respiratory specimens collected between 28 August and 31 December 2014 in BC and tested at the BC PHMRL, 239 (6\%) were positive for EV-D68, representing 218 unique patients ( $n=211$ excluding seven detected also by the SPSN). The majority (172/211; $82 \%)$ had specimens collected between weeks 40 to 45 (early October-early November) (Figure 2) and $72 \%(139 / 194)$ of those with known information were hospitalised.

Based on 2014 BC population estimates [36], incidence of EV-D68 hospitalisations across the 2014 surveillance period was 3 per 100,000 overall and 21, 17, 4 and 1 per 100,000 among those $\langle 5,5-9,10-19$ and $\geq 20$-years-old, with male-to-female ratios of 1.4 overall and 2.1, 1.4, 1.8 and 0.7 by age group, respectively. Hospitalisation rates were essentially unchanged when the period of specimen collection was restricted to 19
September to 31 December 2014, during which screening of respiratory specimens submitted from inpatients of all ages was undertaken: 3 per 100,000 overall and $20,16,4$ and 1 per 100,000 by age category, with maleto-female ratios of 1.4 overall and 2.2, 1.5, 1.4 and 0.7 by age category, respectively.

Between 28 August and 31 October 2014, there were 152 EV-D68 detections by the BC PHMRL. Among these, $146(96 \%)$ case report forms with valid hospitalisation information were submitted to the BCCDC. As shown in Table 3, most of these patients (117/146; $80 \%$ ) were in paediatric age groups with a median age among the 146 cases of eight years (range: 0-90 years). Males were over-represented among paediatric (77/117; 66\%) but not adult $(8 / 29)$ cases. Most cases were hospitalised $(111 / 146 ; 76 \%)$ with a median length-of-stay of three days (range: $1-18$ days) among those patients (106/111; $95 \%)$ discharged at time of reporting. Among the 111 hospitalised patients, nine (8\%) required admission to an intensive care unit (ICU); information on ICU admission was unknown for 15 patients. Asthma history was reported among 47 of 111 (42\%) hospitalised patients and less frequently among non-hospitalised patients (8/35).

\section{Case series of EV-D68-associated neurological or fatal outcomes}

Five EV-D68 cases (paediatric $(n=4)$; male $(n=4)$ ) identified through enhanced passive surveillance in $B C$ were associated with neurological illness including acute flaccid limb weakness $(n=3)$, generalised paralysis $(n=1)$ or head/neck paralysis $(n=1)$ (Table 4$)$ [37]. All had preceding respiratory illness and one had concurrent gastrointestinal illness. All were admitted to hospital; three required ventilation support. None were fatal. All cases were up-to-date for age with polio vaccination. Magnetic resonance imaging in four cases identified hyperintensity predominantly affecting central grey matter of the cervical but also thoracic cord; this was not assessed in Case 4 (adult). Examination of the cerebrospinal fluid (CSF) revealed pleocytosis but normal glucose and, in Cases 1, 2 and 4, elevated protein.

Upper respiratory specimens were EV-D68-positive in all five neurological cases and EV-D68 was also detected by PCR in whole blood from Case 5. All CSF specimens were negative for EV by RT-PCR. One stool specimen was assessed (Case 2) and was EV-D68 negative. Other investigations undertaken at clinician discretion included a range of viral, bacterial and/or fungal pathogens (information available from authors upon request). No alternative aetiologies were identified. Of note, Cases 1, 2 and 5 were Mycoplasma pneumoniae IgM-reactive but, among these, Cases 1 and 2 were $M$. pneumoniae PCR-negative in respiratory specimens and Cases 2 and 5 were $M$. pneumoniae PCRnegative in CSF specimens. Streptococcus pneumoniae was detected by PCR in nasopharyngeal specimens from Cases 3 and 5; however, CSF was PCR-negative in 


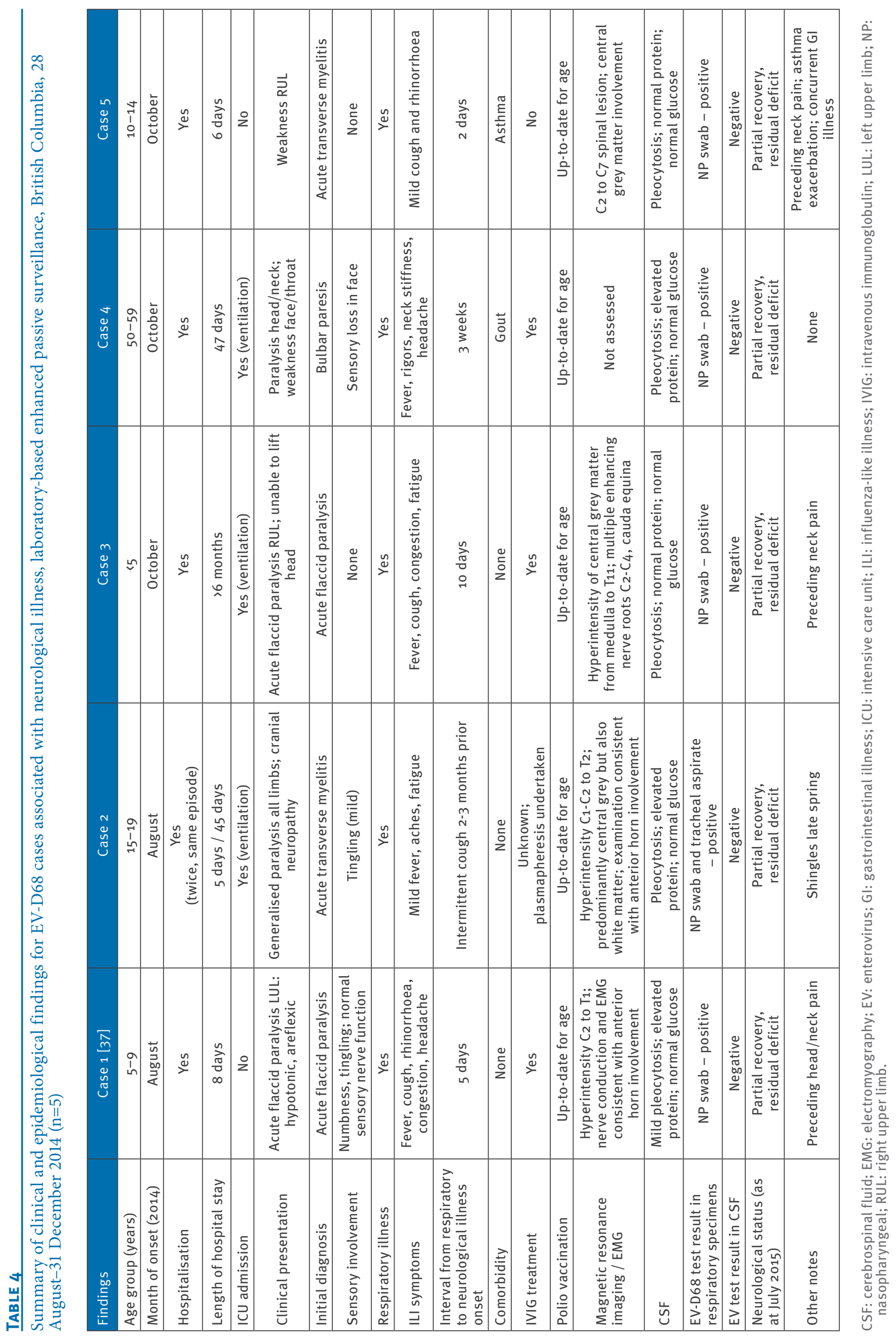


Case 3 and S. pneumoniae was not isolated from CSF of Cases 3 or 5 . As at July 2015 ( $>9-11$ months post onset), all five cases had ongoing neurological deficit.

Three EV-D68 cases in BC with illness onset between August and October 2014 had fatal outcome including: an adult (20-29-years-old) with respiratory failure following acute asthma exacerbation; an adult ( $\geq 65$-yearsold) with multiple comorbidities and respiratory failure following acute chronic obstructive pulmonary disease exacerbation; and a child ( $<5$-years-old) in whom death was ultimately attributed to Group A streptococcal sepsis.

\section{Phylogenetic analysis}

Most $(184 / 187 ; 98 \%)$ of the viruses that were sequenced clustered phylogenetically within clade B (GenBank identifiers: KT873535-KT873716; KT587195-KT587199) [12]. This includes 179 of 182 (98\%) VP1 sequences (including all eight patients with neurological or fatal outcomes) with percent nucleotide identity of 90.6$100 \%$ compared to recent isolates from the US, France, Italy, the Netherlands, and the Philippines (Figure 3). All five VP4/partial VP2 sequences from Alberta also clustered with strains associated with clade B (Figure 4) [12]. Because VP2 sequences from Alberta were partial, clade markers at positions 142-143 described in Lauinger et al. could not be confirmed [7].

Of the clade B VP1 sequences, 8 of 179 (4\%) had a T146S substitution in the immunogenic D-E loop, and two had adjacent asparagine deletions at positions 144-145 in the D-E loop not found in other published sequences; neither the substitution nor the deletion was found in sequences obtained from neurological or fatal cases. Three VP1 sequences from BC, one SPSN specimen from 2013 and two enhanced passive surveillance specimens from 2014, clustered instead in clade $A$ with recent isolates from France, Italy, and the Netherlands, characterised by an asparagine deletion at position 140 in the D-E loop $[7,8]$. Phylogenetic analysis did not suggest clustering by month, severity, inpatient/outpatient status or asthma history.

\section{Discussion}

Canadian investigators used two surveillance approaches to inform risk assessment related to EV-D68-associated illness. These dual surveillance approaches revealed epidemic features of EV-D68 in Canada during the period spanning from August to December 2014. Active sentinel surveillance showed increased EV-D68 detection among outpatient ILI cases affecting all age groups while enhanced passive surveillance showed severe respiratory and neurological disease requiring hospitalisation that occurred at higher incidence, but not exclusively, among children.

The ILI case definition used by the SPSN to standardise outpatient respiratory specimen collection is relatively specific for influenza. It requires fever and cough and at least one other defining symptom such as sore throat [38]. By applying this case definition, we will have missed milder illness caused by other respiratory viruses, for which fever may not be a cardinal feature. Accordingly, the actual number of EV-D68 detections by the SPSN was small $(n=18)$. Among outpatient cases of EV-D68 detected through sentinel surveillance in the Netherlands where an ARI case definition was used, fever or cough were each experienced by 13 of 16 cases and sore throat by 8 of 16 [9]; in Germany, which also used an ARI case definition, 16 of 24 EV-D68 cases experienced fever and cough and 11 of 24 experienced fever, cough and sore throat combined [18]. With comparable community prevalence, community-based sentinel systems that apply a broader ARI case definition will certainly detect more EV-D68 cases. However, sentinel surveillance systems are not intended for the detection of rare events or for quantifying absolute disease burden [39]. They perform best in the detection of highly prevalent conditions and are most valuable for trend analysis. For that purpose, a consistently applied case definition, whether ILI or ARI, is most important. While a single occurrence of a pathogen may reflect chance sporadic detection, multiple case detections across a geographically dispersed network are an indication of widespread community circulation. As such, the eight-fold increase in EV-D68 detection rates by the SPSN in 2014 compared to 2013 , even with an ILI case definition, is indicative of epidemic circulation. Nevertheless, it is acknowledged to be an under-estimate of true incidence.

In Canada, adults $\geq 20$-years-old are predominant SPSN participants ( $>70-75 \%$ [26]) and correspondingly comprised two-thirds of outpatient EV-D68 detections. Among specimens obtained from non-elderly adults 20 to 59-years-old and paediatric cases $<20$-years-old, however, the proportion that tested positive for EV-D68 was equivalent $(4 \%)$. This suggests that children and adults are at comparable risk for outpatient EV-D68 illness that presents as ILI, although there may be differences based on other presentations. The EV-D68 age distribution we describe as extending to adults is similar to outpatient sentinel surveillance observations reported from the Netherlands and Germany $[8,9,18]$. Although few countries outside of Canada or within Europe have used existing sentinel schemes to explore historic and current EV-D68 patterns $[8,9,18]$, such infrastructure could prove highly informative if invoked elsewhere and may also be efficient for characterising other pathogens displaying sudden, unexpected but widespread activity.

Passive surveillance systems are most sensitive to severe disease, particularly if involving children or clusters. Enhanced passive surveillance conducted in $B C$ also reflected this paediatric hospital-based skew, driven by specimen collection largely initiated at clinician discretion and laboratory-confirmation protocols that initially prioritised specimens collected in hospital and from children. All confirmatory testing for EV-D68 in $B C$ was conducted at the BC PHMRL and this served 
as the trigger for enhanced data collection in 2014 . However, from 19 September 2014, all inpatient respiratory specimens submitted to the BC PHMRL were screened for EV-D68, allowing unique populationbased comparison of hospitalisation incidence by age and sex. Ultimately, about three quarters of EV-D68 detections were hospitalised patients among whom more than $80 \%$ were in paediatric age groups, consistent with the age distribution of prior documented outbreaks $[5-7,11,13,15,17]$. While this pattern likely reflects a tip-of-the-iceberg hospital-based surveillance phenomenon, children may indeed be at higher risk owing to greater exposure opportunities and lower likelihood of pre-existing cross-reactive immunity, an immuno-epidemiological hypothesis that still requires evaluation.

The majority of EV-D68 cases were detected in earlyOctober to early-November 2014, also consistent with prior documented outbreaks in North America and Europe [3,8-11,17]. However, labour action among teachers in September 2014 in BC resulted in school closures extending to October 2014 that may have delayed EV-D68 circulation in school-aged children. In order to detect possible seasonal recurrence in 2015, all inpatient and outpatient respiratory specimens collected in BC from 1 August to 29 September 2015 were routinely screened with an EV-D68-specific RT-PCR assay at the BC PHMRL; however, none of 709 specimens screened in 2015 were EV-D68-positive. Conversely, between 28 August and 29 September 2014, 18 EV-D68 cases were already detected, including 13 hospitalisations and two neurological events, reinforcing the exceptional activity in 2014 .

Males were over-represented among paediatric but not adult cases in the 2014 BC enhanced surveillance data, a pattern that has been documented before $[3,6-9,11,17]$, but was neither discernible in the small outpatient sentinel series reported here nor in data from Germany [18]. Male predominance may reflect increased pre-pubertal prevalence of asthma in boys $[40,41]$. Overall $38 \%$ of EV-D68 cases described here reported asthma compared to $7 \%$ of the general $B C$ population [40]. Asthma is a recognised risk factor for severe EV-D68 illness $[1,6,13,15,28]$, although underlying mechanisms are unclear. Other respiratory viruses, notably RVs with which EV-D68 shares biological features, are associated with acute exacerbation and more severe lower respiratory illness in asthmatic individuals. This effect is thought to be mediated through Th2-skewed pro-inflammatory cytokine production and impaired antiviral responses [42,43]. S. pneumoniae was co-detected in upper respiratory specimens from at least 7 of our 111 (6\%) hospitalised EV-D68 cases for whom we had complete enhanced surveillance data. While S. pneumoniae is believed to enhance RV-induced disease severity, including asthma exacerbation [44], our finding likely reflects background carriage rates of S. pneumoniae in children.
EV-D68 was associated with fatal outcome in three BC patients, including two adults with underlying comorbidity and one child in whom bacterial co-infection played a role. Enhanced passive surveillance in $B C$ also detected five cases (four paediatric) of EV-D68associated neurological illness, all with preceding respiratory symptoms and prolonged neurological deficit. The number of EV-D68 cases with neurological manifestations or fatal outcome detected in $B C$ is higher per capita than reported elsewhere during the 2014 epidemic $[20,23,24]$. This likely reflects the centralised, province-wide laboratory screening and enhanced public health follow-up that was undertaken compared to other cluster-driven analyses, although we cannot rule out true regional differences. Other EVs are known causes of neurological disease, particularly poliovirus and EV-A71, but also EV-D94, which is closely related to EV-D68 $[45,46]$. However, only two cases of EV-D68associated neurological illness had been documented globally prior to the 2014 epidemic, both from the US. One involved acute flaccid paralysis in a young adult in 2005 and the second involved a fatal meningomyeloencephalitis in a child in 2008 [3,47]. EV-D68 was detected in the CSF of both these cases $[3,47]$.

Clinical features in our neurological case series are consistent with reports elsewhere in 2014. They include acute flaccid limb weakness, bulbar weakness, and/or cranial nerve dysfunction in association with the detection of EV-D68 in respiratory specimens [1924]. Also similar to other reports, magnetic resonance imaging findings showed grey matter involvement in multiple spinal levels but mostly affecting the cervical spine [19-24]. EV-D68 was detected in whole blood from one $B C$ patient, but in the absence of EV-D68 detection in CSF specimens, a causal role for EV-D68 remains unproven in this case series, as elsewhere in 2014 [19-24]. It should be noted, however, that recovery of poliovirus from the CSF has also only rarely been reported in cases of paralytic poliomyelitis [48]. The finding of detectable M. pneumoniae IgM in three of four neurological cases described here is intriguing given similar serologic findings in a small proportion of other recent EV-D68 neurological case reports $[20,21]$ and the independent association between $M$. pneumoniae and neurological illness [49,50]. Like other recent reports $[20,21]$, however, $M$. pneumoniae was not detected in respiratory or CSF specimens suggesting that antibody findings we report likely reflect prior infection or potentially a false-positive or crossreactive antibody response.

Phylogenetic analysis of EV-D68 viruses from both outpatients and inpatients showed clade B viruses predominated in Canada during the 2014 epidemic period, clustering with recent (2013-2014) sequences from the US and elsewhere but distinct from more historical sequences $[9,12]$. Increased variability in the $V P_{1}$ region, inclusive of the immunogenic $B-C$ and $D-E$ loops, may have enabled EV-D68 to escape antibody recognition $[2,8,9,12,34]$. We did not identify mutations 
or clustering by severe outcome, but full genome analysis would be required to assess other neurotropic or virulence markers, such as in the 5'-untranslated region $[7,12,51]$.

There are limitations to our analyses, foremost related to surveillance methods. Heightened awareness through media and other clinician communications likely influenced patient and provider behaviours related to care-seeking, index of suspicion and testing during the 2014 epidemic period. Nevertheless, neither will the surveillance systems have captured all cases nor will the findings reflect true incidence or disease burden. Small numbers limit our power to test statistical associations. A causal versus contributory or coincidental role for EV-D68 in severe illness cannot be concluded; investigations and their timing were mostly at clinician discretion and other aetiologies, including co-infection, may have been under-recognised. We did not assess other types of EV among EV/RV-positive specimens to compare with the EV-D68 experience. Molecular diagnostic testing for EV-D68 was not routinely performed historically and EV-D68 typing assays were developed and validated real-time in response to the evolving 2014 epidemic, also influencing comparisons across space and time. Laboratory protocols showed comparable performance in a national validation study, and all specimens included in the SPSN analysis were collected within seven days of ILI onset ( $71 \%$ within four days); nevertheless, other variation in specimen collection (e.g. type, viral load), handling, transport and processing may have influenced detection rates between participating provinces. These considerations are, however, relevant to all laboratory-based surveillance. Epidemiological data collection and/or reporting were incomplete particularly when drawn from electronic medical records (as per enhanced passive surveillance) rather than from direct patient/clinician interview (as per active sentinel surveillance). Analyses restricted to patients with known information will have underestimated the proportion with some risk factors/conditions.

Despite these limitations, the dual surveillance approaches we report suggest generalised increase in EV-D68-associated outpatient illness across a broad age distribution during the 2014 epidemic period. Severe respiratory and neurological illness requiring hospitalisation predominantly, but not exclusively, affected children, with possible fatal outcome among those with comorbidity or co-infection. Active surveillance, including both outpatient and inpatient settings, is needed from more areas and additional seasons to further inform EV-D68 incidence, spectrum of illness, and potential at-risk groups for severe or unusual outcomes.

\section{Acknowledgements}

Funding for the Canadian SPSN was provided by the Canadian Institutes of Health Research (grant \# TPA-90193),
British Columbia Centre for Disease Control, Alberta Health and Wellness, Ministère de la santé et des services sociaux du Québec, Institut national de santé publique du Québec, and the Public Health Agency of Canada.

The authors would like to thank the patients and their families who contributed to the SPSN and enhanced passive surveillance data; the public health nurses and epidemiologists in the regional health authorities in $\mathrm{BC}$ who completed the enhanced surveillance case report forms; the sentinel physicians participating in the Canadian SPSN in BC, Alberta, and Quebec; the provincial coordinators for the Canadian SPSN at the BC Centre for Disease Control, TARRANT Viral Watch in Alberta, and the Institut national de santé publique du Quebec; the laboratory technicians who conducted the EV-D68 sequencing and serotyping at the BC Public Health Microbiology and Reference Laboratory, the Alberta Provincial Laboratory (namely Kanti Pabbaraju and Sallene Wong), and the Quebec Provincial Laboratory and the input into EV-D68 surveillance from national partners in other provinces and the Public Health Agency of Canada. We gratefully acknowledge the authors, originating and submitting laboratories of the sequences obtained from GenBank used in phylogenetic analyses.

\section{Conflicts of interest}

MK has received research grants from Roche, Merck, Hologic, Siemens and Boerhinger Ingelheim for unrelated studies. All other authors declare that they have no conflicts of interest.

\section{Authors' contributions}

Conception or design of the work: DMS, CC, GDS, JAD, FRD, TW; data acquisition: DMS, MM, RG, SP, DH, SR, SA, GDS, JAD; data/specimen analysis: DMS, CC, SS, GDS, RT, KF, SJD, CM, PT, MK; interpretation of the data for the work: DMS, CC, SS, MM, RG, SP, DH, SR, SA, GDS, JAD, RT, KF, SJD, CM, PT, MK; drafting the work or revising it critically for important intellectual content: All; approval of manuscript submission: All.

\section{References}

1. ObersteMS, MaherK, SchnurrD, FlemisterMR, LovchikJC, PetersH, et al. Enterovirus 68 is associated with respiratory illness and shares biological features with both the enteroviruses and the rhinoviruses. I Gen Virol. 2004;85(Pt 9):2577-84.DOI: 10.1099/vir.0.79925-o PMID: 15302951

2. ImamuraT, OshitaniH. Global reemergence of enterovirus D68 as an important pathogen for acute respiratory infections. Rev Med Virol. 2015;25(2):102-14.DOI: 10.1002/rmv.1820 PMID: 25471236

3. Centers for Disease Control and Prevention, KhetsurianiN, Lamonte-FowlkesA, OberstS, PallanschMA. Enterovirus surveillance--United States, 1970-2005.MMWR Surveill Summ. 2006;55(8):1-20.PMID: 16971890

4. SchiebleJH, FoxVL, LennetteEH. A probable new human picornavirus associated with respiratory diseases.Am J Epidemiol. 1967;85(2):297-310.PMID: 4960233

5. Centers for Disease Control and Prevention (CDC),. Clusters of acute respiratory illness associated with human enterovirus 68--Asia, Europe, and United States, 2008-2010.MMWR Morb Mortal Wkly Rep. 2011;60(38):1301-4.PMID: 21956405

6. HasegawaS, HiranoR, Okamoto-NakagawaR, IchiyamaT, ShirabeK. Enterovirus 68 infection in children with asthma attacks: virus-induced asthma in Japanese children.Allergy. 2011;66(12):1618-20.DOI: 10.1111/j.1398-9995.2011.02725.X PMID: 21958204

7. LauingerIL, BibleJM, HalliganEP, AaronsEJ, MacMahonE, TongCY. Lineages, sub-lineages and variants of enterovirus 68 in recent outbreaks.PLoS One. 2012;7(4):e36005.DOI: 10.1371/ journal.pone.0036005 PMID: 22536453 
8. MeijerA, van der SandenS, SnijdersBE, Jaramillo-GutierrezG, BontL, van der EntCK, et al. Emergence and epidemic occurrence of enterovirus 68 respiratory infections in The Netherlands in 2010. Virology. 2012;423(1):49-57.DOI: 10.1016/j.virol.2011.11.021 PMID: 22177700

9. MeijerA, BenschopKS, DonkerGA, van der AvoortHG. Continued seasonal circulation of enterovirus D68 in the Netherlands, 2011-2014. Euro Surveill. 2014;19(42):20935.DOI: 10.2807/15607917.ES2014.19.42.20935 PMID: 25358039

10. Rahamat-LangendoenJ, Riezebos-BrilmanA, BorgerR, van der HeideR, BrandenburgA, SchölvinckE, et al. Upsurge of human enterovirus 68 infections in patients with severe respiratory tract infections. J Clin Virol. 2011;52(2):103-6.DOI: 10.1016/j. jcv.2011.06.019 PMID: 21802981

11. RenoisF, BouinA, AndreolettiL. Enterovirus 68 in pediatric patients hospitalized for acute airway diseases.J Clin Microbiol. 2013;51(2):640-3.DOI: 10.1128/JCM.02640-12 PMID: 23224095

12. TokarzR, FirthC, MadhiSA, HowieSR, WuW, SallAA, et al. Worldwide emergence of multiple clades of enterovirus 68. Gen Virol. 2012;93(Pt 9):1952-8.DOI: 10.1099/vir.0.043935-0 PMID: 22694903

13. MidgleyCM, JacksonMA, SelvaranganR, TurabelidzeG, ObringerE, JohnsonD, et al. Severe respiratory illness associated with enterovirus D68 - Missouri and Illinois, 2014. MMWR Morb Mortal Wkly Rep. 2014;63(36):798-9.PMID: 25211545

14. Centers for Disease Control and Prevention (CDC). Enterovirus D68 in the United States, 2014. Atlanta: CDC. [Accessed 3 August 2015]. Available from: http://www.cdc.gov/non-polioenterovirus/about/ev-d68.html

15. BragstadK, JakobsenK, RojahnAE, SkramMK, VainioK, Holberg-PetersenM, et al. High frequency of enterovirus D68 in children hospitalised with respiratory illness in Norway, autumn 2014. Influenza Other Respir Viruses. 2015;9(2):59-63. DOI: 10.1111/irv.12300 PMID: 25534826

16. ESCV-ECDC EV-D68 study group,PoelmanR, Schuffeneckerl, Van Leer-ButerC, JossetL, NiestersHG, LinaB. European surveillance for enterovirus D68 during the emerging NorthAmerican outbreak in 2014.J Clin Virol. 2015;71:1-9.DOI: 10.1016/j.jcv.2015.07.296 PMID: 26364237

17. MidgleySE, ChristiansenCB, PoulsenMW, HansenCH, FischerTK. Emergence of enterovirus D68 in Denmark, June 2014 to February 2015. Euro Surveill. 2015;20(17):21105. Available from: http://www.eurosurveillance.org/ ViewArticle.aspx?Articleld=21105DOI: $10.2807 / 1560-7917$. ES2015.20.17.21105 PMID: 25955773

18. ReicheJ, BöttcherS, DiedrichS, BuchholzU, BudaS, HaasW, et al. Low-level circulation of enterovirus D68-associated acute respiratory infections, Germany, 2014. Emerg Infect Dis. 2015;21(5):837-41.DOI: 10.3201/eid2105.141900 PMID: 25898320

19. Division of Viral Diseases, National Centers for Immunization and Respiratory Diseases, CDC; Division of Vector-Borne Diseases, Division of High-Consequence Pathogens and Pathology, National Center for Emerging and Zoonotic Infectious Diseases, CDC; Children's Hospital Colorado; Council of State and Territorial Epidemiologists, LeshemE. Notes from the field: acute flaccid myelitis among persons aged $\leq 21$ years - United States, August 1-November 13, 2014. MMWR Morb Mortal Wkly Rep. 2015;63(53):1243-4.PMID: 25577990

20. GreningerAL, NaccacheSN, Messacark, ClaytonA, YuG, SomasekarS, et al. A novel outbreak enterovirus D68 strain associated with acute flaccid myelitis cases in the USA (2012-14): a retrospective cohort study. Lancet Infect Dis. 2015;15(6):671-82.DOI: 10.1016/S1473-3099(15)70093-9 PMID: 25837569

21. Centers for Disease Control and Prevention (CDC),AyscueP, Van HarenK, SheriffH, WaubantE, WaldronP, YagiS, et al. . Acute flaccid paralysis with anterior myelitis - California, June 2012 June 2014.MMWR Morb Mortal Wkly Rep. 2014;63(40):9036. Available from: http://www.cdc.gov/mmwr/preview/ mmwrhtml/mm6340a6.htm?s_cid=mm6340a6_wPMID: 25299608

22. Centers for Disease Control and Prevention (CDC), PastulaDM, AliabadiN, HaynesAK, MessacarK, SchreinerT, MaloneyJ, et al. . Acute neurologic illness of unknown etiology in children - Colorado, August-September 2014.MMWR Morb Mortal Wkly Rep. 2014;63(40):901-2.PMID: 25299607

23. LangM, MirandA, SavyN, HenquellC, MaridetS, PerignonR, et al. Acute flaccid paralysis following enterovirus D68 associated pneumonia, France, 2014. Euro Surveill. 2014;19(44):20952. Available from: http://www. eurosurveillance.org/ViewArticle.aspx?Articleld=20952DOI: 10.2807/1560-7917.ES2014.19.44.20952 PMID: 25394254
24. PfeifferHC, BragstadK, SkramMK, DahlH, KnudsenPK, ChawlaMS, et al. Two cases of acute severe flaccid myelitis associated with enterovirus D68 infection in children, Norway, autumn 2014. Euro Surveill. 2015;20(10):21062. Available from: http://www.eurosurveillance.org/ ViewArticle.aspx?Articleld=21062DOI: 10.2807/1560-7917. ES2015.20.10.21062 PMID: 25788251

25. Statistics Canada. Population and dwelling counts, for Canada, provinces and territories, 2011 and 2006 censuses. Ottawa: Statistics Canada; 2015. Available from: http://www12.statcan. gc.ca/census-recensement/2011/dp-pd/hlt-fst/pd-pl/TableTableau.cfm? LANG $=$ Eng $\& T=101 \& S=50 \& O=A$

26. SkowronskiDM, ChambersC, SabaiducS, De SerresG, WinterAL, DickinsonJA, et al. Integrated sentinel surveillance linking genetic, antigenic, and epidemiologic monitoring of influenza vaccine-virus relatedness and effectiveness during the 20132014 influenza season. J Infect Dis. 2015;212(5):726-39.DOI: 10.1093/infdis/jiv177 PMID: 25784728

27. PabbarajuK, WongS, WongAA, TellierR. Detection of enteroviruses and parechoviruses by a multiplex real-time RT-PCR assay.Mol Cell Probes. 2015;29(2):81-5.DOI: 10.1016/j. mcp.2015.02.001 PMID: 25681753

28. DrewsSJ, SimmondsK, UsmanHR, YeeK, FathimaS, TipplesG, et al. Characterization of enterovirus activity, including that of enterovirus D68, in pediatric patients in Alberta, Canada, in 2014. J Clin Microbiol. 2015;53(3):1042-5.DOI: 10.1128/ JCM.02982-14 PMID: 25588657

29. CoirasMT, AguilarJC, GarcíaML, Casası, Pérez-BreñaP. Simultaneous detection of fourteen respiratory viruses in clinical specimens by two multiplex reverse transcription nested-PCR assays.J Med Virol. 2004;72(3):484-95.DOI: 10.1002/jmv.20008 PMID: 14748074

30. PabbarajuK, WongS, ChanENY, TellierR. Genetic characterization of a Coxsackie A9 virus associated with aseptic meningitis in Alberta, Canada in 2010.Virol J. 2013;10(1):93. Available from: http://www.virologyj.com/ content/10/1/93DOI: 10.1186/1743-422X-10-93 PMID: 23521862

31. GregoryJB, LitakerRW, NobleRT. Rapid one-step quantitative reverse transcriptase $P C R$ assay with competitive internal positive control for detection of enteroviruses in environmental samples.Appl Environ Microbiol. 2006;72(6):3960-7.DOI: 10.1128/AEM.02291-05 PMID: 16751503

32. NixWA, ObersteMS, PallanschMA. Sensitive, seminested PCR amplification of VP1 sequences for direct identification of all enterovirus serotypes from original clinical specimens.J Clin Microbiol. 2006;44(8):2698-704.DOI: 10.1128/JCM.00542-06 PMID: 16891480

33. HatchetteTF, DrewsSJ, GrudeskiE, BoothT, MartineauC, DustK, et al. Detection of enterovirus D68 in Canadian laboratories. Clin Microbiol. 2015;53(5):1748-51.DOI: 10.1128/JCM.03686-14 PMID: 25740765

34. ImamuraT, OkamotoM, NakakitaS, SuzukiA, SaitoM, TamakiR, et al. Antigenic and receptor binding properties of enterovirus 68. J Virol. 2014;88(5):2374-84.DOI: 10.1128/JVI.03070-13 PMID: 24371050

35. TamuraK, StecherG, PetersonD, FilipskiA, KumarS. MEGA6: Molecular Evolutionary Genetics Analysis version 6.o. Mol Bio Evol. 2013;30(12):2725-9.DOI: 10.1093/molbev/mst197 PMID: 24132122

36. BC Stats. Population estimates for 2014. Victoria: BC Stats. [Accessed 3 August 2015]. Available from: http:// www.bcstats.gov.bc.ca/StatisticsBySubject/Demography/ PopulationEstimates.aspx

37. SherwoodMD, GanttS, ConnollyM, DobsonS. Acute flaccid paralysis in a child infected with enterovirus D68: a case report.B C Med J. 2014;56:495-8. Accessed 3 August 2015. Available from: http://www.bcmj.org/sites/default/files/ BCMJ_56_Vol10_flaccid_paralysis.pdf

38. JiangL, LeeVJ, LimWY, ChenMI, ChenY, TanL, et al. Performance of case definitions for influenza surveillance. Euro Surveill. 2015;20(22):21145.DOI: 10.2807/1560-7917.ES2015.20.22.21145 PMID: 26062645

39. Centers for Disease Control and Prevention (CDC). Manual for the surveillance of vaccine-preventable diseases. Enhancing Surveillance, Chapter 19. 5 th ed., 2011. Atlanta: CDC. [Accessed 24 September 2015]. Available from: http://www.cdc.gov/ vaccines/pubs/surv-manual/index.html

40. ChenY, JohansenH, ThillaiampalamS, SambellC. Asthma. Statistics Canada Catalogue no. 82-003. Health Rep. 2005;16:43-6.

41. KynykJA, MastronardeJG, McCallisterJW. Asthma, the sex difference.Curr Opin Pulm Med. 2011;17(1):6-11.DOI: 10.1097/ MCP.ob013e3283410038 PMID: 21045697

42. BusseWW, LemanskeRF, GernJE. Role of viral respiratory infections in asthma and asthma exacerbations. Lancet. 
2010;376(9743):826-34.DOI: 10.1016/S0140-6736(10)61380-3 PMID: 20816549

43. JacksonDJ, JohnstonSL. The role of viruses in acute exacerbations of asthma.J Allergy Clin Immunol. 2010;125(6):1178-87, quiz 1188-9.DOI: 10.1016/j. jaci.2010.04.021 PMID: 20513517

44. KloepferKM, LeeWM, PappasTE, KangTJ, VrtisRF, EvansMD, et al. Detection of pathogenic bacteria during rhinovirus infection is associated with increased respiratory symptoms and asthma exacerbations. J Allergy Clin Immunol.

2014;133(5):1301-7, 1307.e1-3.PMID: 24698319

45. Richman DD, Whitley RJ, Hayden FG. Clinical Virology. New York: Churchill Livingston, 1997.

46. SmuraTP, JunttilaN, BlomqvistS, NorderH, KaijalainenS, PaananenA, et al. Enterovirus 94, a proposed new serotype in human enterovirus species D. J Gen Virol. 2007;88(Pt 3):84958.DOI: 10.1099/vir.0.82510-0 PMID: 17325357

47. KreuterJD, BarnesA, McCarthyJE, SchwartzmanJD, ObersteMS Rhodes $\mathrm{CH}$, et al. A fatal central nervous system enterovirus 68 infection. Arch Pathol Lab Med. 2011;135(6):793-6.PMID: 21631275

48. Centers for Disease Control and Prevention (CDC).

Epidemiology and Prevention of Vaccine-Preventable Diseases. Hamborsky J, Kroger A, Wolfe S, eds. 13th ed. Washington D.C. Public Health Foundation, 2015. p. 299. Atlanta: CDC. [Accessed 3 August 2015]. Available from: http://www.cdc.gov/ vaccines/pubs/pinkbook/downloads/polio.pdf

49. KoskiniemiM. CNS manifestations associated with Mycoplasma pneumoniae infections: summary of cases at the University of Helsinki and review.Clin Infect Dis. 1993;17(Suppl 1):S52-7.DOI: 10.1093/clinids/17.Supplement_1.S52 PMID: 8399938

50. HelyMA, WilliamsonPM, TerentyTR. Neurological complications of Mycoplasma pneumoniae infection.Clin Exp Neurol. 1984;20:153-60.PMID: 6439446

51. NorderH, MagniusL. Can sequence data predict enterovirus D68 infection outcome? Lancet Infect Dis. 2015;15(6):620-1.DOI: 10.1016/S1473-3099(15)70107-6 PMID: 25837570 